SPOP
mutations
influence BET
degradation
and sensitivity
to BET
inhibitors
in prostate
cancer

PROSTATE CANCER

\title{
BET inhibitors SPOP right there!
}

Speckle-type POZ protein (SPOP), the substrate-binding subunit of a cullin-3 (CUL3)-based E3 ubiquitin ligase complex, mediates the ubiquitylation and degradation of target proteins. Despite being the most frequently mutated gene in primary prostate cancer, the therapeutic implications of SPOP mutations are incompletely understood. Now, three studies in Nature Medicine identify bromodomain and extraterminal (BET) proteins - oncogenic transcriptional co-activators that are promising targets for epigenetic therapy — as SPOP substrates, and offer mechanistic insights into how SPOP mutations influence BET degradation and sensitivity to BET inhibitors in prostate cancer.

The three groups unanimously reported that wild-type SPOP promotes the ubiquitylation and proteasomal degradation of BET proteins, including bromodomain-containing protein 2 (BRD2), BRD3 and BRD4. Zhang et al. and Dai et al. identified an evolutionarily conserved SPOP-binding motif situated between two BET bromodomains, which was required for SPOP-dependent BET degradation. As prostate cancer-associated SPOP mutations map to the substrate-recognition domain, the groups hypothesized that they might impair BET degradation. Indeed, Zhang et al. and Dai et al. found that SPOP mutants failed to interact with and degrade BET proteins in cell line models, leading to accumulation of BET proteins. Elevated BRD4 levels had pro-tumorigenic effects in vitro and in vivo, and immunohistochemistry analysis demonstrated that BRD4 levels were elevated in $S P O P$-mutated primary prostate cancer specimens, which was associated with poor survival outcomes and progression.

Next, the groups explored whether aberrant BET degradation, conferred by SPOP mutations, could alter sensitivity to BET inhibitors. Zhang et al. reported that expression of the SPOP mutants in cell lines and xenograft mouse models conferred resistance to the anti-growth effects of BET inhibitors JQ1 and I-BET. Similarly, Dai et al. found that SPOP-mutated prostate cancer cell lines and patient-derived organoids were intrinsically resistant to BET inhibitor-induced apoptosis and growth arrest. Importantly, both studies noted that depletion of BET proteins - notably BRD4 — in the SPOP-mutated models resensitized prostate cancer cells to JQ1, demonstrating that stabilization of BET protein levels confers resistance to BET inhibitors. Using transcriptome and BRD4 chromatin immunoprecipitation-sequencing (ChIP-seq) analyses in SPOPmutated prostate cancer cells, Zhang et al. demonstrated that BRD4 accumulation induced upregulation of the GTPase RAC1 and cholesterol biosynthesis pathway genes, which elicit BET inhibitor resistance by activation of AKT-mTOR complex 1 (mTORC1) signalling. Accordingly, coadministration of AKT inhibitor ipatasertib abrogated BET inhibitor resistance.

Intriguingly, Janouskova et al. uncovered a paradox whereby cancer-type specific SPOP mutations that map to the same substrate-recognition domain
confer differential sensitivity to BET inhibitors. Consistent with the other studies, prostate cancer-associated SPOP mutations impaired BET degradation and conferred resistance to JQ1. However, endometrial cancer-associated SPOP mutations enhanced BET degradation and sensitized endometrial cancer cells to JQ1, providing a preclinical rationale for BET inhibitor monotherapy in SPOP-mutant endometrial cancer.

Collectively, the findings provide a rationale for the evaluation of SPOP mutations or BET protein levels as predictive biomarkers to guide BET inhibitor therapy in prostate cancer. Furthermore, detection of cancer-type specific SPOP mutations could be used to select patients who might benefit from BET inhibitor therapy.

Conor A. Bradley

\section{ORIGINAL ARTICLES}

Janouskova, H. et al. Opposing effects of cancertype-specific SPOP mutants on BET protein degradation and sensitivity to BET inhibitors. Nat.Med.http://dx.doi.org/10.1038/nm.4372 (2017)|Dai, X. et al. Prostate cancer-associated SPOP mutations confer resistance to BET inhibitors through stabilization of BRD4. Nat. Med. http://dx.doi.org/10.1038/nm.4378 (2017)| Zhang, P. et al. Intrinsic BET inhibitor resistance in SPOP-mutated prostate cancer is mediated by BET protein stabilization and AKT-mTORC1 activation. Nat. Med. http://dx.doi.org/10.1038/ nm.4379 (2017) 